\title{
Integral equation algorithm for fluids of fully anisotropic molecules
}

F. Lado

Department of Physics, North Carolina State University, Raleigh, North Carolina 27695-8202

E. Lomba

Instituto de Química Física Rocasolano, CSIC, Serrano 119, E-28006 Madrid, Spain

M. Lombardero

Instituto de Química Física Rocasolano, CSIC, Serrano 119, E-28006 Madrid, Spain and Depto. Química

Física I, U. Complutense, E-28040 Madrid, Spain

(Received 23 February 1995; accepted 2 May 1995)

We outline a practical algorithm for the solution of liquid-state integral equations for fluids of fully anisotropic rigid molecules requiring three Euler angles for their configurational description and leading to pair functions of five angular variables. The method is suitable for all potentials. We illustrate the technique with sample results for $\mathrm{SO}_{2}$. () 1995 American Institute of Physics.

The central task in the integral equation approach to the theory of liquids ${ }^{1}$ is the numerical solution of two coupled, nonlinear equations for the direct and indirect correlation functions, $c(12)$ and $\gamma(12)$ : the Ornstein-Zernike equation

$$
\gamma(12)=\frac{\rho}{8 \pi^{2}} \int d \mathbf{3}[c(13)+\gamma(13)] c(32)
$$

and the closure relation

$$
c(12)=\exp [-\beta u(12)+\gamma(12)+B(12)]-1-\gamma(12) .
$$

Here $u(12)$ is the interaction between molecules 1 and 2, $B(12)$ the so-called bridge function (because of the resemblance of its first density diagram to the Wheatstone bridge circuit), $\rho$ the number density, and $\beta=1 / k_{B} T$ the inverse temperature. Various closures result from the choice made for approximating $B(12)$; for the discussion below, we shall assume that $B(12)$ is given. In Eq. (1), the integral is over the coordinates $\mathbf{r}_{3}$ and $\omega_{3}$ of the molecular center and orientation, respectively.

The fivefold angular dependence of the pair functions, $X(12)=X\left(r_{12}, \theta_{1}, \theta_{2}, \phi_{12}, \chi_{1}, \chi_{2}\right)$, for liquids of rigid, fully anisotropic molecules makes these equations excessively complex for numerical work. The first and essential ingredient for their reduction is a spherical harmonic expansion of the correlation functions, ${ }^{2,3}$

$$
\begin{aligned}
X(12)= & \sum_{l_{1} l_{2} m n_{1} n_{2}}\left[\left(2 l_{1}+1\right)\left(2 l_{2}+1\right)\right]^{1 / 2} \\
& \times X_{l_{1} l_{2} m}^{n_{1} n_{2}}\left(r_{12}\right) D_{m n_{1}}^{l_{1}}\left(\omega_{1}\right)^{*} D_{\bar{m} n_{2}}^{l_{2}}\left(\omega_{2}\right)^{*},
\end{aligned}
$$

where the orientations $\omega=(\phi, \theta, \chi)$, the Euler angles, are here referred to the axial line $\mathbf{r}_{12}$ between molecular centers, $D_{m n}^{l}(\omega)$ is a generalized spherical harmonic in the convention of Rose, ${ }^{4}$ and $\bar{m}=-m$. Inversion of the expansion gives the coefficients as

$$
\begin{aligned}
X_{l_{1} l_{2} m}^{n_{1} n_{2}}\left(r_{12}\right)= & \frac{\left[\left(2 l_{1}+1\right)\left(2 l_{2}+1\right)\right]^{1 / 2}}{64 \pi^{4}} \\
& \times \int d \omega_{1} d \omega_{2} X(12) D_{m n_{1}}^{l_{1}}\left(\omega_{1}\right) D_{\overline{m n}_{2}}^{l_{2}}\left(\omega_{2}\right) .
\end{aligned}
$$

With these definitions, the $n_{1}=n_{2}=0$ coefficients, $X_{l_{1} l_{2} m}^{00}(r)$, coincide with those commonly used for fluids of linear molecules. ${ }^{5,6}$

The computational premise then is that a relative few axial coefficients $\gamma_{l_{1} l_{2} m}^{n_{1} n_{2}}(r)$ will suffice for reconstruction of the full angular description $\gamma(12)$. Other quantities, such as the pair distribution function,

$$
g(12)=\exp [-\beta u(12)+\gamma(12)+B(12)],
$$

are obtained from $\gamma(12)$.

A related treatment was proposed by Fries and Patey ${ }^{7}$ and subsequently applied to polar fluids composed of spherically shaped molecules. ${ }^{8}$ The particular treatment of the closure (5) used in Refs. 7 and 8 to avoid multidimensional integration is however unsuitable for strongly anisotropic short range interactions like those that give rise to the molecular shape. ${ }^{9}$

\section{GENERAL PROCEDURE}

With these preliminaries, the iterative procedure now followed in solving Eqs. (1) and (2) is a straightforward generalization of that for linear molecules. ${ }^{6}$ We sketch here the steps in the procedure and elaborate only on the new elements.

(1) $\gamma_{l_{1} l_{2} m}^{n_{1} n_{2}}(r) \rightarrow c_{l_{1} l_{2} m}^{n_{1} n_{2}}(r)$ :closure equation. With a finite set of coefficients $\gamma_{l_{1} l_{2} m}^{n_{1} l_{2}}(r)$ in hand, an iteration for an improved set begins with the construction of $\gamma(12)$ using Eq. (3). The direct correlation function $c(12)$ follows from Eq. (2) and its coefficients $c_{l_{1} l_{2} m}^{n_{1} n_{2}}(r)$ are then computed with Eq. (4). This is by far the most time-consuming step of the calculation and merits special attention. Several techniques to speed up this part are discussed below. 
(2) $\quad c_{l_{1} l_{2} m}^{n_{1} n_{2}}(r) \rightarrow \tilde{c}_{l_{1} l_{2} m}^{n_{1} n_{2}}(k)$ : Fourier transforms. The Ornstein-Zernike (OZ) equation is most conveniently solved in Fourier transform representation. To this end, the spacefixed coefficients

$$
c\left(r ; l_{1} l_{2} l_{1} n_{2}\right)=\sum_{m} C\left(l_{1} l_{2} l ; m \bar{m} 0\right) c_{l_{1} l_{2} m}^{n_{1} n_{2}}(r)
$$

are obtained by a Clebsch-Gordon (CG) transformation and used to generate the Hankel transforms

$$
\tilde{c}\left(k ; l_{1} l_{2} \ln _{1} n_{2}\right)=4 \pi i^{l} \int_{0}^{\infty} d r r^{2} c\left(r ; l_{1} l_{2} \ln _{1} n_{2}\right) j_{l}(k r),
$$

where $j_{l}(x)$ is a spherical Bessel function of order $l$. The transforms for $l>0$ are reduced to sine or cosine transforms by "lowering" $c\left(r ; l_{1} l_{2} l_{n} n_{2}\right)$ to $l=0$ or -1 for $l$ even or odd, respectively. ${ }^{6}$ An inverse CG transformation then yields the axial transform coefficients

$$
\tilde{c}_{l_{1} l_{2} m}^{n_{1} n_{2}}(k)=\sum_{l} C\left(l_{1} l_{2} l ; m \bar{m} 0\right) \tilde{c}\left(k ; l_{1} l_{2} \ln _{1} n_{2}\right)
$$

needed for the OZ equation. In these expressions, $C\left(l_{1} l_{2} l ; m_{1} m_{2} m\right)$ is a Clebsch-Gordon coefficient.

(3) $\tilde{c}_{l_{1} l_{2} m}^{n_{1} n_{2}}(k) \rightarrow \tilde{\gamma}_{l_{1} l_{2} m}^{n_{1} n_{2}}(k)$ : OZ equation. Fourier transformation of the $\mathrm{OZ}$ equation and expansion of the transforms as in Eq. (3) leads to ${ }^{2,3}$

$$
\begin{aligned}
\tilde{\gamma}_{l_{1} l_{2} m}^{n_{1} n_{2}}(k)= & \rho \sum_{l_{3}, n_{3}}(-1)^{m+n_{3}}\left[\tilde{c}_{l_{1} l_{3} m}^{n_{1} n_{3}}(k)\right. \\
& \left.+\tilde{\gamma}_{l_{1} l_{3} m}^{n_{1} n_{3}}(k)\right] \tilde{c}_{l_{3} l_{2} m}^{\bar{n}_{3} n_{2}}(k),
\end{aligned}
$$

from which the $\gamma_{l_{1} l_{2} m}^{n_{1} n_{2}}(k)$ are extracted by matrix operations.

(4) $\tilde{\gamma}_{l_{1} l_{2} m}^{n_{1} n_{2}}(k) \rightarrow \gamma_{l_{1} l_{2} m}^{n_{1} n_{2}}(r)$ : inverse Fourier transforms. The iteration is completed by the analog of step (2), beginning with a CG transformation to space-fixed coefficients,

$$
\tilde{\gamma}\left(k ; l_{1} l_{2} \ln _{1} n_{2}\right)=\sum_{m} C\left(l_{1} l_{2} l ; m \bar{m} 0\right) \tilde{\gamma}_{l_{1} l_{2} m}^{n_{1} n_{2}}(k),
$$

followed by an inverse Hankel transform,

$$
\gamma\left(r ; l_{1} l_{2} \ln _{1} n_{2}\right)=\frac{1}{2 \pi^{2} i^{l}} \int_{0}^{\infty} d k k^{2} \tilde{\gamma}\left(k ; l_{1} l_{2} l n_{1} n_{2}\right) j_{l}(k r) .
$$

This becomes an inverse sine or cosine transform for the "lowered" coefficients, which are then "raised" to the final $l>0$ values. ${ }^{6}$ Another inverse CG transformation,

$$
\gamma_{l_{1} l_{2} m}^{n_{1} n_{2}}(r)=\sum_{l} C\left(l_{1} l_{2} l ; m \bar{m} 0\right) \gamma\left(r ; l_{1} l_{2} l_{1} n_{2}\right),
$$

yields the new axial coefficients of $\gamma(12)$ and completes one iteration. Here the new and old $\gamma(12)$ coefficients are compared; iterations continue until $\gamma(12)$ is adequately selfconsistent.

\section{ACCELERATORS}

The calculations of step (1) above are computationally intensive; we have used several methods to speed them up. The first of these introduces an alternative step (1), labeled step (5), and leads to a new inner loop for the iterations.

(5) $\gamma_{l_{1} l_{2} m}^{\mathrm{LIN} n_{1} n_{2}}(r) \rightarrow c_{l_{1} l_{2} m}^{\mathrm{LIN} n_{1} n_{2}}(r)$ : linearized closure. The greatest improvement is achieved by avoiding step (1) altogether, as proposed recently by Labík et al. $;^{10}$ instead, the coefficients $c_{l_{1} l_{2} m}^{\mathrm{LIN} n_{2} n_{2}}(r)$ are obtained from the linearized closure

$$
c^{\mathrm{LIN}}(12)=c(12)+H(12)\left[\gamma^{\mathrm{LIN}}(12)-\gamma(12)\right],
$$

where $H(12)=\partial c(12) / \partial \gamma(12)$. The precise form of $H(12)$ depends on the closure used; for the HNC results discussed below, it is $H(12)=g(12)-1$. The computational cost now comes in evaluating the coefficients for a product function $A(12)=B(12) C(12),^{3}$

$$
\begin{aligned}
A_{l_{1} l_{2} m}^{n_{1} n_{2}}(r)= & \sum_{l_{1}^{\prime} l_{2}^{\prime} m^{\prime} n_{1}^{\prime} n_{2}^{\prime}} \sum_{l_{1}^{\prime \prime} l_{2}^{\prime \prime} m^{\prime \prime} n_{1}^{\prime \prime} n_{2}^{\prime \prime}} B_{l_{1}^{\prime} l_{2}^{\prime} m^{\prime}}^{n_{1}^{\prime} n^{\prime}}(r) C_{l_{1}^{\prime \prime} l_{2}^{\prime \prime} m^{\prime \prime}}^{n_{1}^{\prime \prime} n^{\prime \prime}} \\
& \times\left[\frac{\left(2 l_{1}^{\prime}+1\right)\left(2 l_{1}^{\prime \prime}+1\right)\left(2 l_{2}^{\prime}+1\right)\left(2 l_{2}^{\prime \prime}+1\right)}{\left(2 l_{1}+1\right)\left(2 l_{2}+1\right)}\right]^{1 / 2} \\
& \times C\left(l_{1}^{\prime} l_{1}^{\prime \prime} l_{1} ; m^{\prime} m^{\prime \prime} m\right) C\left(l_{1}^{\prime} l_{1}^{\prime \prime} l_{1} ; n_{1}^{\prime} n_{1}^{\prime \prime} n_{1}\right) \\
& \times C\left(l_{2}^{\prime} l_{2}^{\prime \prime} l_{2} ; \bar{m}^{\prime} \bar{m}^{\prime \prime} \bar{m}\right) C\left(l_{2}^{\prime} l_{2}^{\prime \prime} l_{2} ; n_{2}^{\prime} n_{2}^{\prime \prime} n_{2}\right) .
\end{aligned}
$$

This cost will normally be rather less than that of step (1), but still not inconsequential.

The iterations now run through an inner loop of relabeled steps (2)-(5) for the $\gamma^{\mathrm{LIN}}(12)$ coefficients, keeping $\gamma(12)$ and $c(12)$ fixed:

(2) $c_{l_{1} l_{2} m}^{\mathrm{LIN}_{1} n_{2}}(r) \rightarrow \tilde{c}_{l_{1} l_{2} m}^{\mathrm{LIn} n_{1} n_{2}}(k)$ : Fourier transforms;

(3) $\tilde{c}_{l_{1} l_{2} m}^{\mathrm{LIN} n_{1} n_{2}}(k) \rightarrow \tilde{\gamma}_{l_{1} l_{2} m}^{\mathrm{LIN} n_{1} n_{2}}(k):$ OZ equation;

(4) $\tilde{\gamma}_{l_{1} l_{2} m}^{\mathrm{LIN} n_{1} n_{2}}(k) \rightarrow \gamma_{l_{1} l_{2} m}^{\mathrm{LIN} n_{1} n_{2}}(r)$ : inverse transforms;

(5) $\gamma_{l_{1} l_{2} m}^{\mathrm{LIN} n_{1} n_{2}}(r) \rightarrow c_{l_{1} l_{2} m}^{\mathrm{LIN}_{1} n_{2}}(r)$ :linearized closure.

When self-consistency, tested after step (4), is achieved for $\gamma^{\mathrm{LIN}}(12), \gamma(12)$ is updated as $\gamma^{\mathrm{LIN}}(12)$ and the program returns to step (1) for a full recalculation of the $c(12)$ coefficients, whereupon the inner loops begins anew, starting from $\gamma^{\mathrm{LIN}}(12)=\gamma(12)$ and $c^{\mathrm{LIN}}(12)=c(12)$. The calculation terminates when $\gamma(12)$ reaches self-consistency in the outer loop.

When step (1) is performed, the application of the full quadrature is restricted by two bounds in $r$ : (i) within a predetermined core region, $r<r_{\text {core }}$, the coefficients of $g(12)$ are simply set to zero and (ii) beyond a cutoff separation, $r>r_{\text {cutoff }}$, determined dynamically by the magnitude of the anisotropic part of $\gamma(12)$, they are obtained from a secondorder expansion of the anisotropic part of the exponent in Eq. (5). This again involves the product algorithm of Eq. (14).

Finally, when the full quadrature is performed, for $r_{\text {core }}<r<r_{\text {cutoff }}$, its execution is accelerated by a generalized fast-transform algorithm proposed by Orszag. ${ }^{11}$ We first rewrite Eq. (3) in terms of $x_{1}=\cos \theta_{1}, \quad x_{2}=\cos \theta_{2}$, $y=\cos \phi_{12}, \quad z_{1}=\cos \chi_{1}, \quad$ and $\quad z_{2}=\cos \chi_{2}, \quad$ so that $\gamma(12)=\gamma\left(r, x_{1} x_{2}, y, z_{1} z_{2}\right)$. The discrete version evaluates 
$\gamma(12)$ at the points $x_{i_{1}}, x_{i_{2}}, y_{j}, z_{k_{1}}, z_{k_{2}}$ where the $x_{i}$ are the $\nu$ roots of the Legendre polynomial $P_{\nu}(\cos \theta), y_{j}$ of the Chebyshev polynomial $T_{\nu}(\cos \phi)$, and $z_{k}$ of the Chebyshev polynomial $T_{\nu}(\cos \chi)$. Equation (3) then reads

$$
\begin{aligned}
\gamma\left(r, i_{1}, i_{2}, j, k_{1}, k_{2}\right)= & \sum_{n_{2}, n_{1}, m=-M}^{M} \sum_{l_{2}=L_{2}}^{M} \sum_{l_{1}=L_{1}}^{M} \gamma_{l_{1} l_{2} m}^{n_{1} n_{2}}(r) \\
& \times \hat{d}_{m n_{1}}^{l_{1}}\left(i_{1}\right) \hat{d}_{\bar{m} n_{2}}^{l_{2}}\left(i_{2}\right) e_{m}(j) \\
& \times e_{n_{1}}\left(k_{1}\right) e_{n_{2}}\left(k_{2}\right),
\end{aligned}
$$

where we have put $\hat{d}_{m n}^{l}(x)=(2 l+1)^{1 / 2} d_{m n}^{l}(\theta), \quad e_{m}(y)$ $=\exp (i m \phi)$, and $e_{n}(z)=\exp (i n \chi)$; in the summation limits, we use $L_{\alpha}=\max \left(m, n_{\alpha}\right), \alpha=1,2$. For brevity, we have further written $i_{1}$ for $x_{i_{1}}, i_{2}$ for $x_{i_{2}}$, etc.; $M$ and $\nu$ are parameters of the calculation. Equation (15) constitutes a separable, five-dimensional transform; to rapidly evaluate this expression, the five-dimensional transform is replaced by five one-dimensional transforms:

$$
\begin{aligned}
& \gamma_{l_{2} m}^{n_{1} n_{2}}\left(r, i_{1}\right)=\sum_{l_{1}=L_{1}}^{M} \gamma_{l_{1} l_{2} m}^{n_{1} n_{2}}(r) \hat{d}_{m n_{1}}^{l_{1}}\left(i_{1}\right), \\
& \gamma_{m}^{n_{1} n_{2}}\left(r, i_{1}, i_{2}\right)=\sum_{l_{2}=L_{2}}^{M} \gamma_{l_{2} m}^{n_{1} n_{2}}\left(r, i_{1}\right) \hat{d}_{\bar{m} n_{2}}^{l_{2}}\left(i_{2}\right), \\
& \gamma^{n_{1} n_{2}}\left(r, i_{1}, i_{2}, j\right)=\sum_{m=-M}^{M} \gamma_{m}^{n_{1} n_{2}}\left(r, i_{1}, i_{2}\right) e_{m}(j) \\
& \gamma^{n_{2}}\left(r, i_{1}, i_{2}, k_{1}\right)=\sum_{n_{1}=-M}^{M} \gamma^{n_{1} n_{2}}\left(r, i_{1}, i_{2}, j\right) e_{n_{1}}\left(k_{1}\right) \\
& \gamma\left(r, i_{1}, i_{2}, j, k_{1}, k_{2}\right)=\sum_{n_{2}=-M}^{M} \gamma^{n_{2}}\left(r, i_{1}, i_{2}, j, k_{1}\right) e_{n_{2}}\left(k_{2}\right) .
\end{aligned}
$$

Operations involving the $e_{m}(y)$ and $e_{n}(z)$ basis functions are done in complex arithmetic; the final result is of course real. The sum of operations in Eqs. (16) is asymptotically smaller than that of Eq. (15) and the procedure thus constitutes a "fast separable transform". 11

Evaluation of the fivefold integral in Eq. (4) using Gaussian quadrature,

$$
\begin{aligned}
c_{l_{1} l_{2} m}^{n_{1} n_{2}}(r)= & w^{3} \sum_{i_{1}, i_{2}, j, k_{1}, k_{2}=1}^{\nu} w_{i_{1}} w_{i_{2}} c\left(r, i_{1}, i_{2}, j, k_{1}, k_{2}\right) \\
& \times \hat{d}_{m n_{1}}^{l_{1}}\left(i_{1}\right) \hat{d}_{\bar{m} n_{2}}^{l_{2}}\left(i_{2}\right) e_{\bar{m}}(j) e_{\bar{n}_{1}}\left(k_{1}\right) e_{\bar{n}_{2}}\left(k_{2}\right)
\end{aligned}
$$

proceeds through a similar sequence of one-dimensional transforms in reverse order and requires the same number of operations. In Eq. (17), the $w_{i}=1 /\left(1-x_{i}^{2}\right)\left[P_{\nu}^{\prime}\left(x_{i}\right)\right]^{2}$ are the weights for Gauss-Legendre quadrature, while $w=1 / \nu$ is the constant weight for Gauss-Chebyshev quadrature.

For simplicity, Eqs. (15)-(17) have been written for the implicit ranges $0<\phi_{12}, \chi_{1}, \chi_{2}<\pi$. If a full range 0 to $2 \pi$ is needed, appropriate modifications are made.

The iterative scheme described above, referred to as Picard iteration, is often only slowly convergent. Successful hybrids of the much more powerful (but also computationally more costly) Newton-Raphson iteration with the Picard

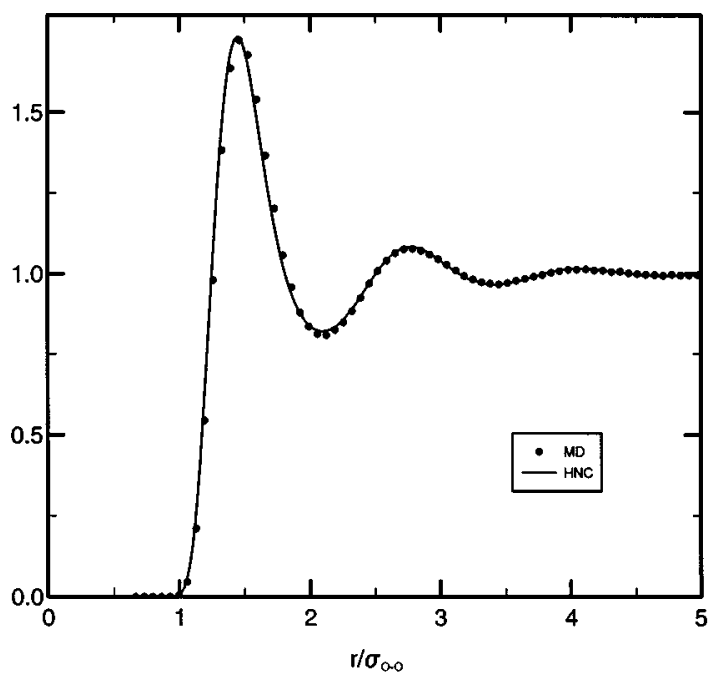

FIG. 1. Radial coefficient of the molecular pair distribution function for fluid $\mathrm{SO}_{2}$ : HNC integral equation vs molecular dynamics results.

method have been developed for simple liquids ${ }^{12-16}$ and recently generalized for liquids of linear molecules. ${ }^{10,17,18}$ These same generalizations can be used for the present nonlinear-molecule calculation. In the calculations reported below we used $\mathrm{LMV}^{13}$ acceleration, but for just the radial component $\tilde{\gamma}_{000}^{00}(k)$. A broader implementation is being prepared.

Finally, we have used Ng's method ${ }^{19}$ to construct at each iteration a "best guess" for the next $\gamma_{l_{1} l_{2} m}^{n_{1} n_{2}}(r)$, based on the previous three iterates, to further accelerate convergence, as well as an iterate-mixing scheme ${ }^{20}$ to smooth out the iterations.

\section{RESULTS}

Here we present some preliminary results for a model of nonlinear polyatomic molecules intended to reproduce the

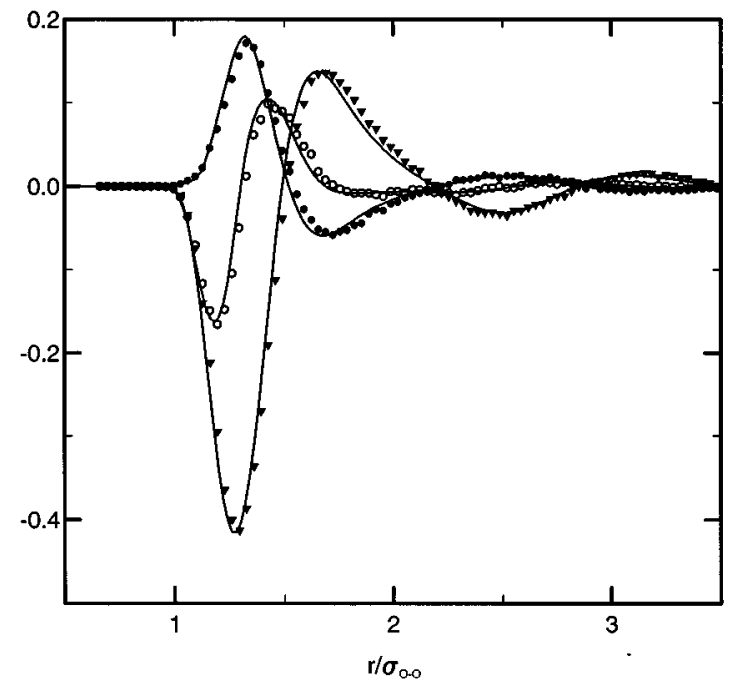

FIG. 2. Angular projections of the molecular pair distribution function for fluid $\mathrm{SO}_{2}$. Symbols denote MD results: open circles correspond to the $g_{100}^{00}(r)$ projection, filled circles to $g_{200}^{00}(r)$, and filled triangles to $g_{200}^{20}(r)$. The HNC data are presented as solid curves. 
properties of fluid $\mathrm{SO}_{2}$. Specifically, we use the model proposed by Sokolic et al. ${ }^{21}$ in a molecular dynamics study of liquid $\mathrm{SO}_{2}$. For simplicity, of the model variations studied in Ref. 21 , we consider here only the uncharged model $B$, a site-site potential with three interaction sites per molecule. The molecular geometry is defined by the apex angle $\theta_{\mathrm{O}-\mathrm{S}-\mathrm{O}}=119.5^{\circ}$ and the bond length $r_{\mathrm{S}-\mathrm{O}}=1.434 \AA$. We have chosen the interaction site potential parameters for thermodynamic state 4 in Ref. 21 , i.e., $\sigma_{\mathrm{SS}}=3.625 \AA$, $\sigma_{\mathrm{OO}}=3.015 \AA$, $\epsilon_{\mathrm{SS}} / k_{B}=167.5 \mathrm{~K}$, and $\epsilon_{\mathrm{OO}} / k_{B}=66.8 \mathrm{~K}$. Cross interaction parameters are determined according to Lorentz-Berthelot rules. The molar volume is set to $V_{m}=53.21 \mathrm{~cm}^{3} / \mathrm{mol}(\rho$ $=1.204 \mathrm{~g} / \mathrm{cm}^{3}$ ) and the temperature to $793 \mathrm{~K}$. (Note that this is a much higher temperature than that of thermodynamic state 4 in Ref. 21.) In this preliminary work we restrict ourselves to the hypernetted-chain (HNC) approximation.

The integral equation was solved with 49 angular coefficients up to $g_{333}^{33}$; as a benchmark, we carried out a microcanonical molecular dynamics simulation with a 500 particle sample that equilibrated at $T=793 \pm 3 \mathrm{~K}$. The $\mathrm{HNC}$ solution yields a compressibility factor $\beta P^{\mathrm{HNC}} / \rho=3.78$, which is in reasonable agreement with our simulation value $\beta P^{\mathrm{MD}} / \rho=3.02$. The HNC prediction for the configurational energy is $\beta U^{\mathrm{HNC}} / N=-2.17$, also quite close to the simulation result $\beta U^{\mathrm{MD}} / N=-2.24$. In accord with these thermodynamic agreements, the microscopic structure of the fluid is also reliably reproduced by the integral equation. This can be appreciated in Figs. 1 and 2, which depict the center-tocenter distribution function and three of the low-order angular projections of the molecular distribution function. Bearing in mind the usually poor performance of the $\mathrm{HNC}$ closure for molecular systems, ${ }^{22}$ these results can be considered remarkably good.

\section{ACKNOWLEDGMENTS}

E. L. and M. L. acknowledge the support of the Spanish Dirección General de Investigación Científica y Técnica (DGICYT) under Grant No. PB91-0110. Dr. D. Martín is acknowledged for his invaluable technical assistance in the development of the Molecular Dynamics program.

${ }^{1}$ J. P. Hansen and I. R. McDonald, Theory of Simple Liquids (Academic, New York, 1986).

${ }^{2}$ L. Blum and A. J. Torruella, J. Chem. Phys. 56, 303 (1972); L. Blum, ibid. 57, 1862 (1972).

${ }^{3}$ C. G. Gray and K. E. Gubbins, Theory of Molecular Liquids (Clarendon, Oxford, 1984), Vol. 1.

${ }^{4}$ M. E. Rose, Elementary Theory of Angular Momentum (Wiley, New York, 1957).

${ }^{5}$ W. B. Streett and K. E. Gubbins, Annu. Rev. Phys. Chem. 28, 373 (1977).

${ }^{6}$ F. Lado, Mol. Phys. 47, 283, 299 (1982).

${ }^{7}$ P. H. Fries and G. N. Patey, J. Chem. Phys. 82, 429 (1985).

${ }^{8}$ P. G. Kusalik and G. N. Patey, Mol. Phys. 65, 1105 (1988).

${ }^{9}$ See J. A. Anta, E. Lomba, C. Martín, M. Lombardero, and F. Lado, Mol. Phys. 84, 743 (1995) for a hybrid method that implements both the FriesPatey expansion of the closure and direct multidimensional integration applied to Lennard-Jones diatomic fluids.

${ }^{10}$ S. Labík, R. Pospíśil, and A. Malijevský, J. Comput. Phys. 115, 12 (1994).

${ }^{11}$ S. A. Orszag, in Science and Computers, edited by G.-C. Rota (Academic, New York, 1986).

${ }^{12}$ M. J. Gillan, Mol. Phys. 38, 1781 (1979).

${ }^{13}$ S. Labík, A. Malijevský, and P. Voňka, Mol. Phys. 56, 709 (1985).

${ }^{14}$ G. Zerah, J. Comput. Phys. 61, 280 (1985).

${ }^{15}$ M. Kinoshita and M. Harada, Mol. Phys. 65, 599 (1988).

${ }^{16}$ E. Lomba, Mol. Phys. 68, 87 (1989).

${ }^{17}$ P. H. Fries and M. Cosnard, J. Phys. (Paris) 48, 723 (1987).

${ }^{18}$ M. Kinoshita and M. Harada, Mol. Phys. 74, 443 (1991).

${ }^{19}$ K. Ng, J. Chem. Phys. 61, 2680 (1974).

${ }^{20}$ A. A. Broyles, J. Chem. Phys. 33, 456 (1960).

${ }^{21}$ F. Sokolić, Y. Guissani, and B. Guillot, Mol. Phys. 56, 239 (1985).

${ }^{22}$ M. Lombardero, C. Martín, and E. Lomba, J. Chem. Phys. 97, 2724 (1992). 